\title{
In vitro and in vivo effects of suloctidil on growth and biofilm formation of the opportunistic fungus Candida albicans
}

\author{
Beini Zeng ${ }^{1}$, Jiachen Li ${ }^{1}$, Yajie Wang ${ }^{1}$, Pengxiang Chen ${ }^{2}$, Xiaohong Wang ${ }^{1}$, Jianfeng \\ Cui ${ }^{3}$, Lidong Liu ${ }^{4}$, Xiaoyan Hu${ }^{1}$, Qian $\mathrm{Cao}^{1}$, Ying Xiao ${ }^{1}$, Junlu Dong ${ }^{5}$, Yundong Sun ${ }^{1}$ \\ and Yabin Zhou ${ }^{1}$ \\ ${ }^{1}$ Department of Pathogenic Biology and Key Laboratory for Experimental Teratology of Chinese Ministry of Education, School \\ of Basic Medical Sciences, Shandong University, Jinan, Shandong 250012, People's Republic of China \\ ${ }^{2}$ Department of Radiation Oncology, Qilu Hospital of Shandong University, Jinan, Shandong 250012, People's Republic of \\ China \\ ${ }^{3}$ Department of Urology, Qilu Hospital of Shandong University, Jinan, Shandong 250012, People's Republic of China \\ ${ }^{4}$ Department of Obstetrics and Gynecology, Qilu Hospital of Shandong University, Jinan, Shandong 250012, People's Republic \\ of China \\ ${ }^{5}$ Department of Neurobiology, School of Basic Medical Sciences, Shandong University, Jinan, Shandong 250012, People's \\ Republic of China \\ Correspondence to: Yabin Zhou, email: zyb@sdu.edu.cn \\ Yundong Sun, email: syd@sdu.edu.cn \\ Keywords: C. albicans, biofilm, suloctidil, virulence, vaginal candidiasis \\ Received: December 22, $2016 \quad$ Accepted: June 19, $2017 \quad$ Published: July 25, 2017 \\ Copyright: Zeng et al. This is an open-access article distributed under the terms of the Creative Commons Attribution License 3.0 \\ (CC BY 3.0), which permits unrestricted use, distribution, and reproduction in any medium, provided the original author and source \\ are credited.
}

\section{ABSTRACT}

As the most frequent fungal pathogen in humans, Candida albicans can develop serious drug resistance because its biofilms are resistant to most antifungal agents; this leads to an urgent need to develop novel antifungals. Here, we evaluated the efficacy of an antithrombotic drug, suloctidil, against $C$. albicans biofilms in vitro and in vivo. We found that suloctidil is effective to inhibit $C$. albicans biofilm, with a minimum inhibitory concentration ( $\mathrm{MIC}_{80}$ ) of $4 \mathrm{\mu g} / \mathrm{mL}$, a biofilm inhibiting concentration $\left(\mathrm{BIC}_{80}\right.$ ) of $16 \mu \mathrm{g} / \mathrm{mL}$ and a biofilm eradicating concentration $\left(B \mathrm{CC}_{80}\right.$ ) of $64 \mu \mathrm{g} / \mathrm{mL}$. Furthermore, the concentration-dependent characteristics of suloctidil were shown by its time-kill curves. Scanning electron microscopy images clearly revealed the morphological effects of suloctidil on biofilm. Yeast-to-hyphal form switching is a key virulence factor of $C$. albicans; therefore, we performed hyphal growth tests and observed that suloctidil inhibited yeast-to-hyphal form switching. This result was consistent with the downregulation of hypha-specific gene ( $H W P 1, A L S 3$, and ECE1) expression levels after suloctidil treatment. In vivo, $256 \mu \mathrm{g} / \mathrm{mL}$ of suloctidil significantly reduced fungal counts $(P<0.01)$ compared to that in groups without treatment; the treatment group induced a slight histological reaction, especially when the treatment lasted for 5 days $(P<0.01)$. Taken together, our data suggest that suloctidil is a potential antifungal agent.

\section{INTRODUCTION}

Candida. albicans is an opportunistic pathogen found in approximately $30 \%-70 \%$ of individuals; this pathogen asymptomatically colonizes the skin, mouth, vaginal mucosa, and gastrointestinal tract and causes candidiasis [1-3]. According to the Centers for Disease Control, oral candidiasis develops in approximately $7 \%$ of infants, $31 \%$ of AIDS patients, and $20 \%$ of cancer patients receiving chemotherapy [4]. Vulvovaginal candidiasis is more common and is estimated to occur more than once in $75 \%$ of healthy women [5]. Invasive candidiasis has a high mortality of $40 \%-60 \%$ [6] because C. albicans adapts to various environmental conditions by developing biofilms, which encase themselves in a self-released slimy polysaccharide and protein layer 
Table 1: Antifungal activity of suloctidil against planktonic cells and biofilms of C. albicans strains YEM30 and LC3

\begin{tabular}{lccc}
\hline Drug concentration and strain & $\mathrm{MIC}_{80}(\boldsymbol{\mu g} / \mathbf{m L})$ & $\mathbf{B I C}_{80}(\mu \mathrm{g} / \mathrm{mL})$ & $\mathbf{B E C}_{80}(\mu \mathrm{g} / \mathrm{mL})$ \\
\hline YEM30 & 4 & 16 & 64 \\
LC3 & 4 & 16 & 64 \\
\hline
\end{tabular}

Antifungal activity of suloctidil against planktonic cells and biofilms of C. albicans strains YEM30 and LC3.

that enables $C$. albicans to adhere to surfaces [6-8]. The formation of $C$. albicans biofilms includes adhesion, cell growth, initial colonization, and maturation phases [8]. Biofilm formation is an important virulence factor of $C$. albicans and occurs in host tissues, prostheses, and indwelling medical devices, such as urinary and vascular catheters, (dental) implants, and heart valves [6, 9-11]. The biofilm significantly increases the resistance of $C$. albicans to traditional antifungal drugs, biofilm-associated C. albicans is 4000 times more resistant to fluconazole than $C$. albicans in its planktonic form $[6,12]$. Hence, alternatives to conventional antifungal agents are urgently needed to damage $C$. albicans biofilms.

Suloctidil (1-(4-isopropylthiophenyl)-2-noctylaminopropanol) is a vascular antispasmodic and antithrombotic drug [13] with in vitro antifungal properties against $C$. albicans [14]. However, the activity of suloctidil against $C$. albicans biofilms has not been studied. The present study aims to explore the inhibitory effect of suloctidil on the formation of $C$. albicans biofilm and on preformed biofilm to evaluate its potential therapeutic application in biofilm-associated candidiasis.

\section{RESULTS}

\section{Antifungal activity of suloctidil}

$\mathrm{MIC}_{80}$ is defined as the lowest concentration of suloctidil that inhibited $80 \%$ of cell growth compared to the control (without suloctidil). The $\mathrm{MIC}_{80}$ of YEM30 and LC30 was $4 \mu \mathrm{g} / \mathrm{mL}$ (Table 1). A higher concentration completely inhibited their growth.

\section{Inhibition of suloctidil on biofilm formation and preformed biofilm}

The effect of suloctidil on biofilm formation was evaluated by $\mathrm{BIC}_{80}$ (biofilm inhibiting concentration), which is defined as the lowest concentration of suloctidil that inhibited $80 \%$ of the metabolic activity of the biofilm formation compared with the control. We found that the $\mathrm{BIC}_{80}$ of YEM30 and LC30 was $16 \mu \mathrm{g} /$ $\mathrm{mL}$ (Table 1, Figure 1). The effect of suloctidil on the preformed biofilm was evaluated by $\mathrm{BEC}_{80}$ (biofilmeradicating concentration), which is defined as the lowest concentration of suloctidil that eradicated $80 \%$ of the biofilm compared to the control. The $\mathrm{BEC}_{80}$ for YEM30 and LC30 was $64 \mu \mathrm{g} / \mathrm{mL}$ (Table 1, Figure 1).

\section{Time-kill curve}

To further study the kinetics of the anti-biofilm activity of the drugs, we performed the time-kill curve assay, which provides growth kinetic information over time and a more detailed picture of the effects of drugs on cell viability. Thus, the time-kill curve assay significantly enhances our understanding of the dynamic relationships between antifungals and C. albicans $[24,26]$. Suloctidil exhibited dose-dependent activity against the two strains (Figure 2). In YEM30, the inhibitory activity of suloctidil against biofilm formation weakened the exposure over 6 $\mathrm{h}$ for sub-BIC ( $4 \mu \mathrm{g} / \mathrm{mL})$ and $12 \mathrm{~h}$ for sub-BIC $(8 \mu \mathrm{g} / \mathrm{mL})$ (Figure 2A), respectively, and $6 \mathrm{~h}$ for both sub-BIC $(4 \mu \mathrm{g} /$ $\mathrm{mL}$ and $8 \mu \mathrm{g} / \mathrm{mL}$ ) in LC3 (Figure 2B). Suloctidil (16 and $32 \mu \mathrm{g} / \mathrm{mL}$ ) completely inhibited biofilm formation, and the fungicidal endpoint for YEM30 and LC3 was achieved after $3 \mathrm{~h}$ at BIC $(16 \mu \mathrm{g} / \mathrm{mL})$ and $2 \times \mathrm{BIC}(32 \mu \mathrm{g} / \mathrm{mL})$ of suloctidil (Figure 2A and 2B).

In addition, suloctidil $(64,128$, and $256 \mu \mathrm{g} / \mathrm{mL})$ rapidly exerted its activity against the mature-phase biofilm in both strains. Less than $0.5 \mathrm{~h}$ was needed to arrive at the fungicidal endpoint (Figure 2C and 2D), and the inhibitory activity of suloctidil against the preformed biofilm weakened the exposure at $0.5 \mathrm{~h}$ for sub-BEC (32 $\mu \mathrm{g} / \mathrm{mL}$ ) in YEM30 (Figure $2 \mathrm{C}$ ) and $3 \mathrm{~h}$ for sub-BEC (32 $\mu \mathrm{g} / \mathrm{mL}$ ) in LC3 (Figure 2D).

\section{Scanning electron microscopy}

SEM images are shown in Figure 3. Compared with the control group (Figure 3A and 3D), the biofilm exposed to $64 \mu \mathrm{g} / \mathrm{mL}$ of suloctidil for $1 \mathrm{~h}$ (Figure $3 \mathrm{~B}$ and $3 \mathrm{E})$ showed slightly shrunken budding cells. After $24 \mathrm{~h}$, the treated biofilm (Figure 3C and 3F) showed obvious cellular damage: cells were completely shriveled, and budding cells had fallen off the hyphae.

\section{Hyphal growth test}

In the control group (Figure 4A), after 12 hours of growth, Candida albicans hyphae were clearly visible. If suloctidil $(16 \mu \mathrm{g} / \mathrm{mL})$ was added at the beginning of the 
A
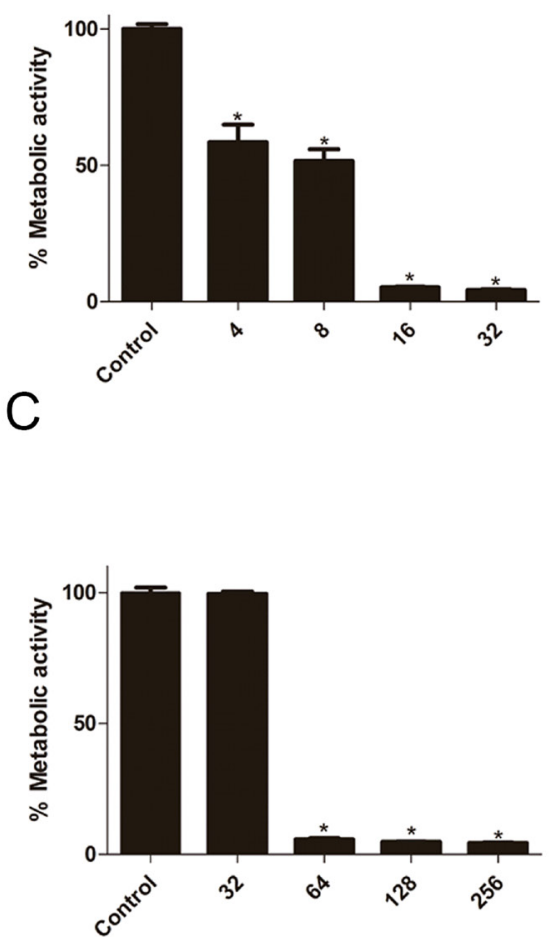

B
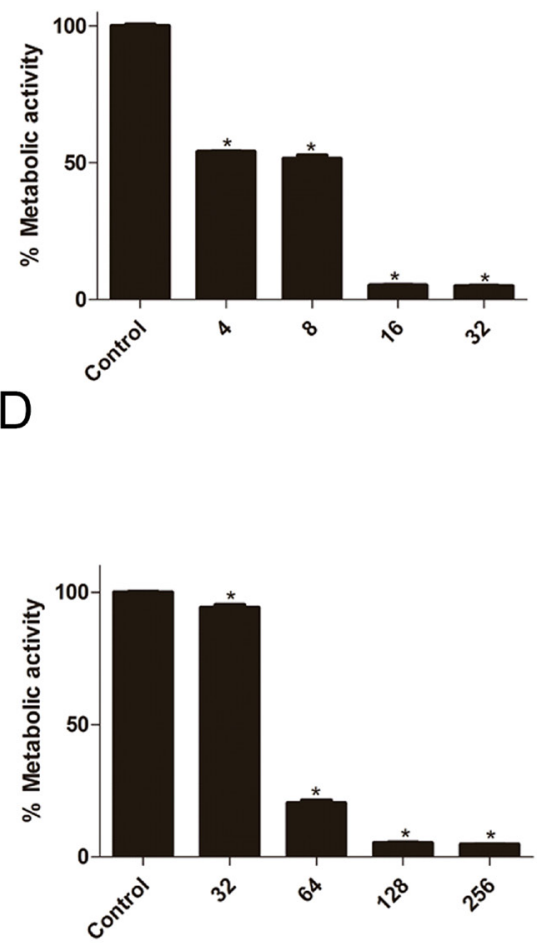

Figure 1: Effect of suloctidil on $C$. albicans biofilms. Effect of various suloctidil concentrations on biofilm formation of $C$. albicans YEM30 (A) and LC3 (B). Effect of suloctidil on preformed biofilms of YEM30 (C) and LC3 (D). The untreated group was set as the control. $* P<0.05 ; * * \mathrm{P}<0.01$.
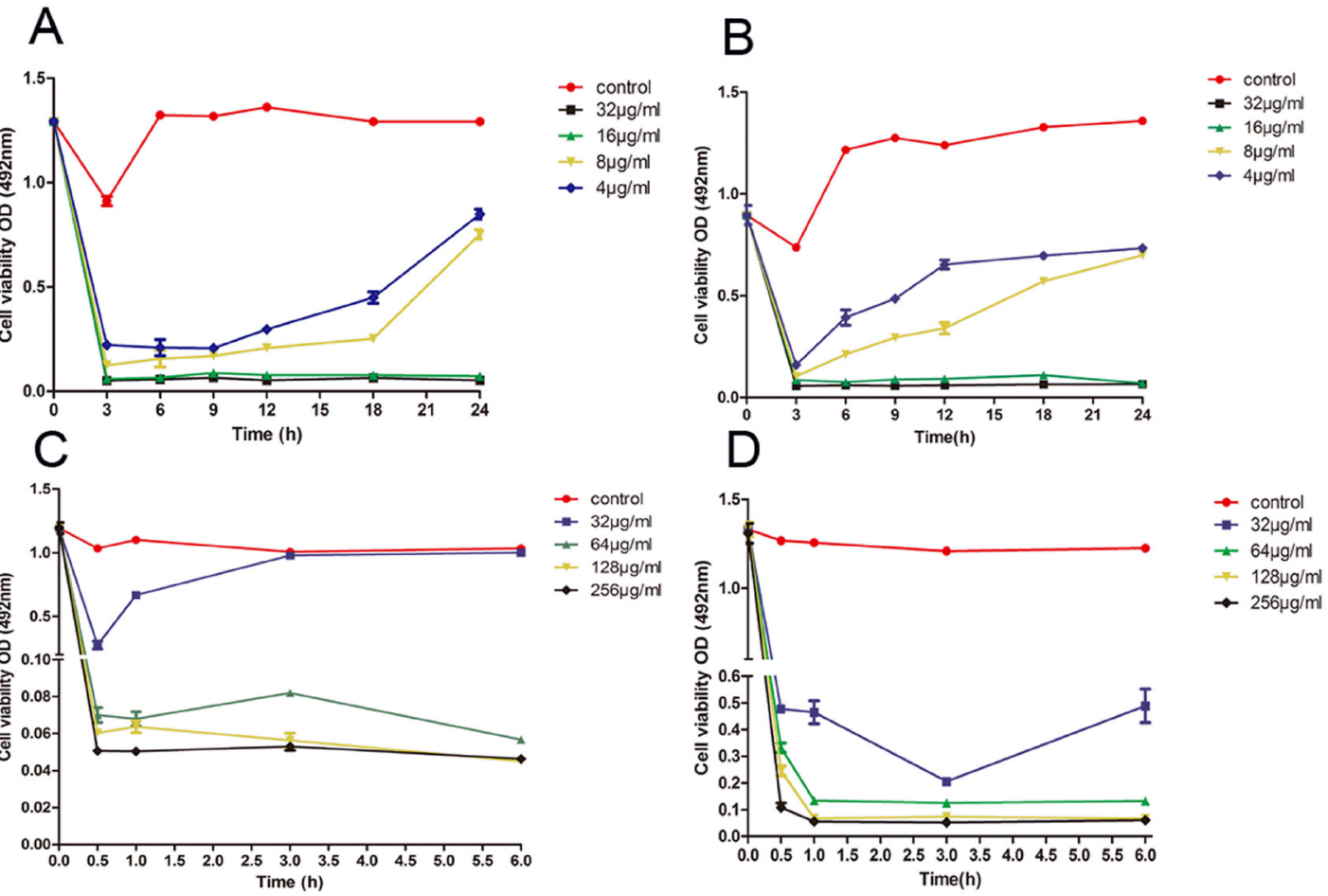

Figure 2: Time-kill curve of suloctidil against C. albicans biofilms. Representative time-kill curves of $4 \mu \mathrm{g} / \mathrm{ml}, 8 \mu \mathrm{g} / \mathrm{ml}, 16 \mu \mathrm{g} / \mathrm{ml}$, and $32 \mu \mathrm{g} / \mathrm{ml}$ suloctidil on biofilm formation of C. albicans YEM30 (A) and LC3 (B). Representative time-kill curves of $32 \mu \mathrm{g} / \mathrm{mL}, 64 \mu \mathrm{g} /$ $\mathrm{mL}, 128 \mu \mathrm{g} / \mathrm{mL}$, and $256 \mu \mathrm{g} / \mathrm{mL}$ suloctidil on preformed biofilms of YEM30 (C) and LC3 (D). The untreated group was used as the control. 
C. albicans culture, its hypha was completely suppressed (Figure 4B); if antibiotics were added after 2 hours of Candida albicans culture, only a very small amount of hyphae were observed (Figure 4C). However, suloctidil $(16 \mu \mathrm{g} / \mathrm{mL})$ could not decrease hypha growth after four hours of $C$. albicans culture (Figure 4D).

\section{Relative expression of $C$. albicans hyphal specific genes}

The expression levels of hypha-specific genes, Hyphal Wall Protein 1 (HWP1) [6], Agglutinin Like Sequence 3 ( $A L S 3$ ) [24], and extent of cell elongation 1 (ECE1) [25], in suloctidil-treated cells were significantly reduced by 2.8-fold, 2.3-fold, and 4.9-fold, respectively, compared with those in the control (Figure 5). This result also revealed that suloctidil down-regulated certain hyphaspecific genes (HWP1, ALS3, and ECE1) of C. albicans.

\section{In vivo therapeutic efficacy of suloctidil for C. albicans infections}

The in vivo antifungal efficacy of suloctidil was further investigated in a murine model of vaginal candidiasis. The results in Figure 6 show a remarkable reduction in the $C$. albicans load of mice treated with suloctidil compared with that of control (PBS)-treated mice, beginning at 5 days post-infection $(P<0.05)$. This
A

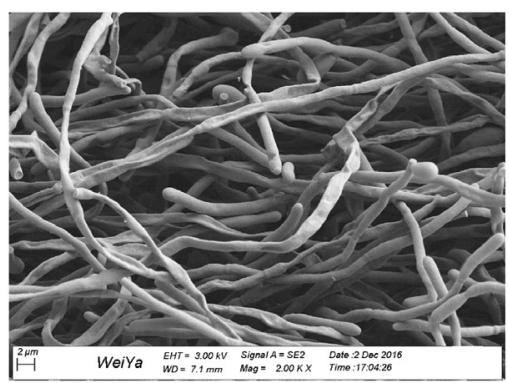

D

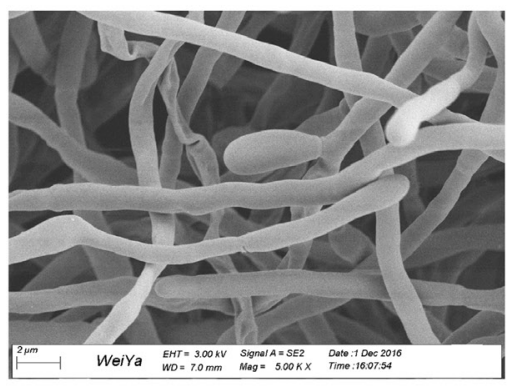

B

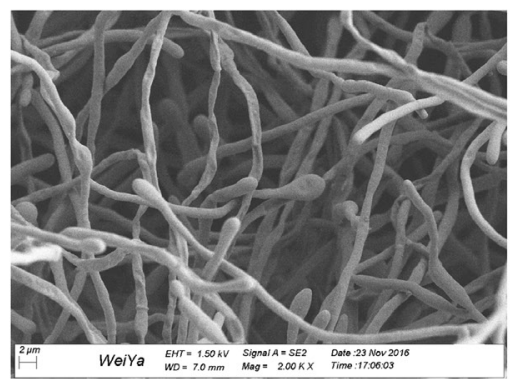

E

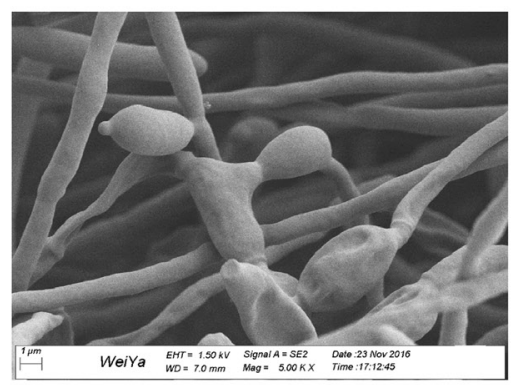

C

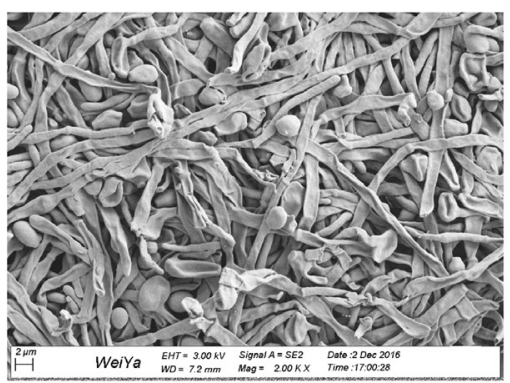

$\mathrm{F}$

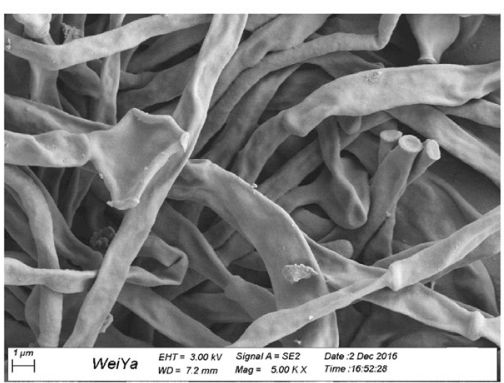

Figure 3: Scanning electron microscopy images of $\boldsymbol{C}$. albicans biofilms. Images of preformed biofilms of $C$. albicans YEM30 treated with suloctidil $(64 \mu \mathrm{g} / \mathrm{mL})$ for $1 \mathrm{~h}(\mathbf{B}, \mathbf{E})$ and $24 \mathrm{~h}(\mathbf{C}, \mathbf{F})$. The untreated group was used as the control (A, D).

A

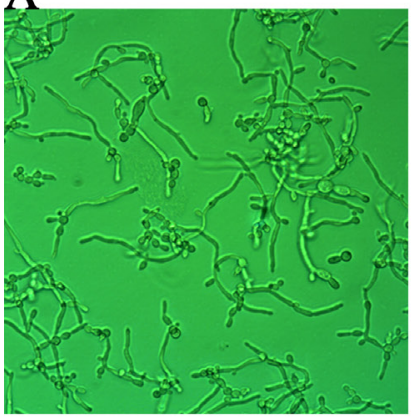

B

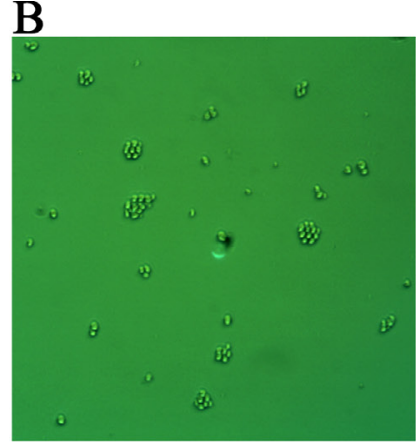

C

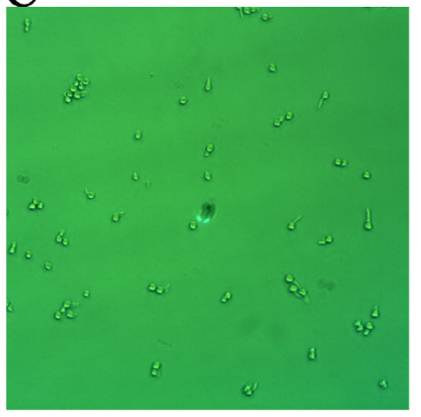

D

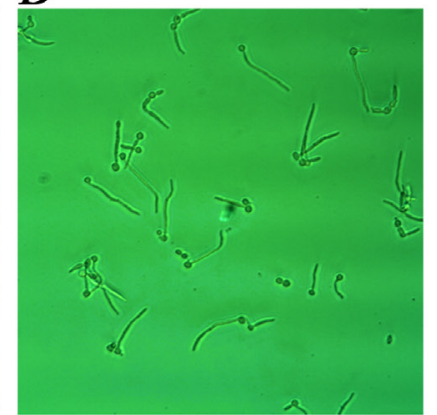

Figure 4: Morphological changes of $C$. albicans after suloctidil treatment. (A) Control; morphological changes of $C$. albicans at different culture times (0 hour (B), 2 hrs (C) and 4 hrs (D)) exposed to suloctidil $(16 \mu \mathrm{g} / \mathrm{ml})$. C. albicans was cultured for 12 hrs. 
beneficial effect was maintained until 15 days postinfection, especially at the 9 th $(P<0.01)$ and 15 th days $(P<0.01)$.

\section{Suloctidil-treated mice show reduced inflammatory pathologic changes}

We compared the histopathological changes in the vaginal tissue between mice with or without suloctidil treatment after infection. At day 5 post-infection, large amounts of cellular infiltration comprising primarily neutrophils and mononuclear cells were found in the mucosa propria and submucosa in the vehicle-treated infected mice; the suloctidil-treated mice showed undetected inflammatory cell infiltration (Figure 7; $P<0.05)$. At day 9 post-infection, vehicle-treated infected mice showed significant cellular infiltration, comprising primarily lymphocyte and mononuclear cells in the mucosa propria, and the suloctidil-treated mice showed mild histologic changes (Figure 7). Both groups showed

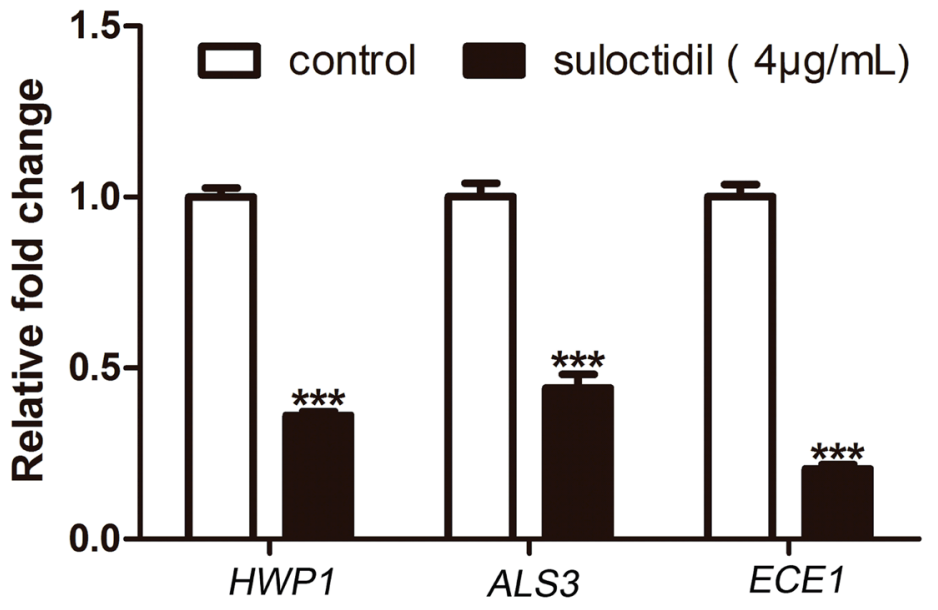

Figure 5: qRT-PCR analysis of expression of hypha-specific genes (HWP1, ALS3, and ECE1) in C. albicans treated with suloctidil. $(4 \mu \mathrm{g} / \mathrm{mL})$ for 6 hours $* P<0.05 ; * * P<0.01 ; * * * P<0.001$.

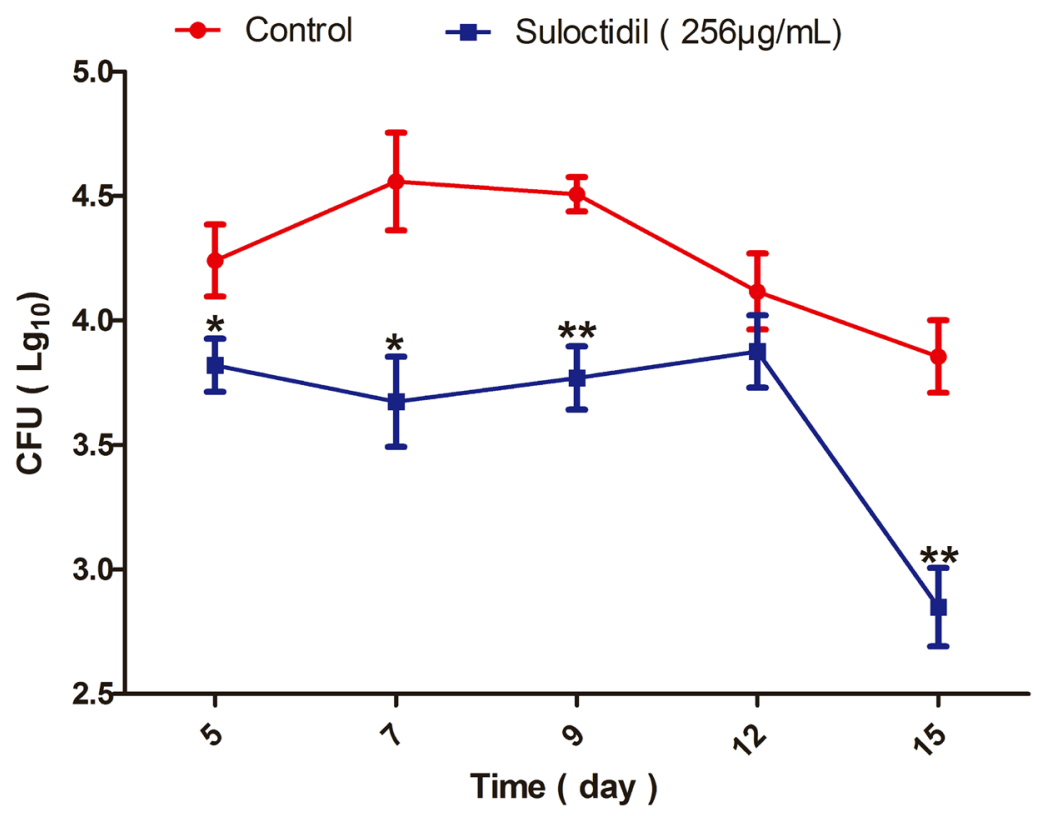

Figure 6: Measurement of $\boldsymbol{C}$. albicans load in mice treated with suloctidil. Vaginal lumen of mice under pseudoestrus condition were infected with C. albicans YEM30 and treated with suloctidil $(256 \mu \mathrm{g} / \mathrm{mL})$ every day. At 5, 7, 9, 12 and 15 days post-infection, the fungal burden of vaginal lavage fluids was collected and determined via the colony forming units (CFU) assay. A significant difference was observed between the suloctidil-treated group and the vehicle-treated control group $(P<0.05)$. Data are the mean $\pm \mathrm{SD}(\mathrm{n}=$ five mice) for one representative experiment in three independent experiments. $* P<0.05$, $* * P<0.01$. 
inflammation at day 15 post-infection; however, the suloctidil-treated mice showed less lymphocyte and cellular infiltration in the mucosa propria (Figure 7).

\section{DISCUSSION}

Biofilm formation is a crucial virulence factor of C. albicans that is closely associated with its pathogenesis $[10,26]$. The development of $C$. albicans biofilms includes three phases: the adhesion phase $(0-1.5 \mathrm{~h})$, the initial colonization phase $(1.5-25.5 \mathrm{~h})$, and the maturation phase (25.5-19.5 h) [8]. C. albicans biofilms formed under clinical conditions have intrinsic resistance to antifungal agents [26].

Previous studies have shown that suloctidil is a calcium-channel blocker [27]. The current study found that suloctidil has antifungal activity and synergy with FLU against $C$. neoformans [14]. However, the inhibitory activity of suloctidil against $C$. albicans biofilms has not been studied. In the present study, the anti-biofilm
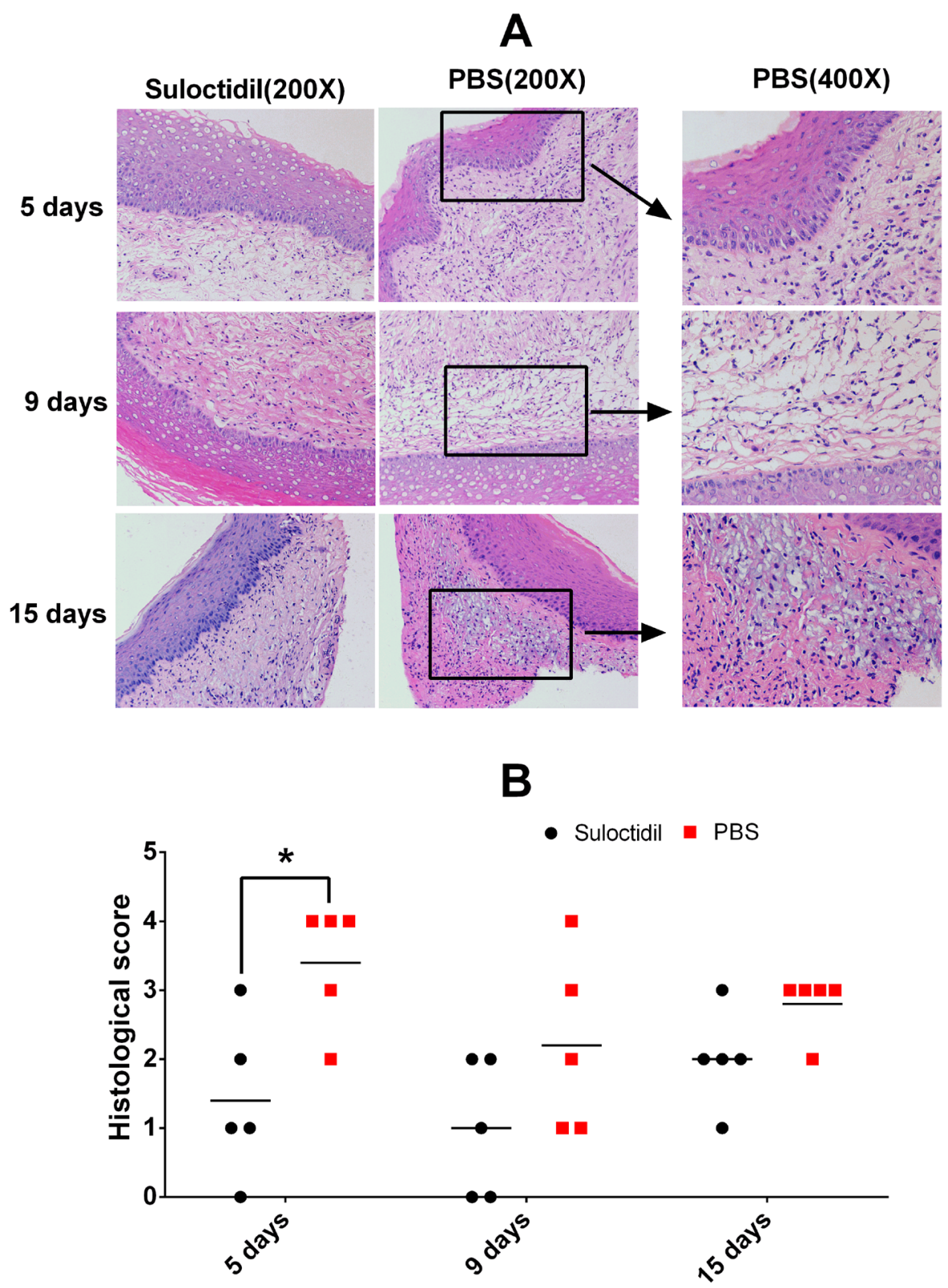

Figure 7: Suloctidil-treated mice show reduced inflammatory pathological changes. Vaginal tissues of mice pretreated with suloctidil or vehicle were collected after infection and analyzed for histological changes by hematoxylin and eosin staining under light microscopy. Representative images (Magnification: 200x, 400x) are shown in (A), and areas of lymphocytic inflammation in each section are marked by rectangles. Histopathology scores for vaginal tissues from Kunming mice are shown in (B) $(\mathrm{n}=5$ /group, 7-8 weeks old). $* P<0.05$. 
Table 2: Primer lists of $A C T 1, H W P 1, A L S 3, E C E 1$ for qRT-PCR

\begin{tabular}{llll}
\hline Primer & Sequence (5'-3') & Primer & Sequence (5'-3') \\
\hline$A C T 1$-f & GGTTTGGAAGCTGCTGGTATTGACC & $A C T 1-\mathrm{r}$ & ACGTTCAGCAATACCTGGGAACATG \\
$H W P 1$-f & TGGTGCTATTACTATTCCGG & $H W P 1-\mathrm{r}$ & CAATAATAGCAGCACCGAAG \\
$A L S 3-\mathrm{f}$ & TACTTCCACAGCTGCTTCCA & $A L S 3-\mathrm{r}$ & GGAGCATTACCACCACCATT \\
$E C E 1-\mathrm{f}$ & GCTGGTATCATTGCTGATAT & $E C E 1-\mathrm{r}$ & GGAGCATTACCACCACCATT \\
\hline
\end{tabular}

activities of suloctidil in vitro and in vivo were examined for the first time. The XTT assay results and SEM images showed significant reduction in metabolic activities and morphological changes of $C$. albicans after suloctidil treatment.

Based on the inhibitory testing results, when 16 $\mu \mathrm{g} / \mathrm{mL}$ of suloctidil was administered at the initial phase, almost all $C$. albicans stayed in the yeast phase without transforming to the hyphal phase, which suggested that suloctidil inhibited hyphal growth. Hyphae are a characteristic feature and constitute the structure of biofilm. Thus, hyphal formation and adherence are vital and essential for biofilm development and sustainability [28]. Furthermore, the morphological transition from yeast to hyphae is strongly associated with fungal infection, and the change from the yeast phase to filamentous phase is critical in the pathogenesis of fungi [29]. A previous study showed that $C$. albicans without hyphae-forming ability is non-virulent in mouse models and displays weaker pathogenicity [30]. Thus, hyphal formation and growth, as well as the yeast-hyphae transition phase, are highly relevant to $C$. albicans biofilm formation and pathogenicity. Therefore, suloctidil has an important effect on the treatment of $C$. albicans infection.

The expression profile of hyphal growth-associated genes (HWP1, ALS3, and ECE1) was analyzed to gain insights into the molecular mechanism of suloctidilmediated hyphal growth inhibition. Expression profile analysis revealed that suloctidil (4 $\mu \mathrm{g} / \mathrm{mL})$ downregulated the expression of hyphal genes, which inhibited hyphal growth. HWP1 is a well-characterized hypha-specific gene that mediates cell-cell interactions and improves the biofilm formation of C. albicans [31, 32]. Moreover, $H W P 1$ regulates the adhesion of $C$. albicans to epithelial cells via the Hwp1 protein [6]. $E C E 1$ is a hypha-induced gene that is related to cell elongation [31, 33]. ALS3 is a member of the agglutininlike sequence $(A L S)$ gene family that encodes cell-wall glycoproteins [24]. ALS3 contributes to cell adhesion, whereas both $A L S$ and $H W P 1$ genes are highly expressed in hyphae and play essential roles in the yeast-to-hypha morphological transition of C. albicans [24, 34-37]. The suloctidil-mediated down-regulation of hypha-related genes may be a cause of mycelial growth inhibition, perhaps sufficient even to prevent the biofilm formation of $C$. albicans.
In conclusion, the effect of suloctidil against $C$. albicans biofilm was evaluated in vitro and in vivo. A low dose of suloctidil ( $4 \mu \mathrm{g} / \mathrm{mL}$ ) inhibited the yeast-tohypha morphological transition of $C$. albicans as well as the expression of $H W P 1, A L S 3$, and ECE1 genes. Suloctidil suppressed the formation of $C$. albicans biofilms by down-regulating the expression of $H W P 1$, $A L S 3$, and ECE1 to prevent the morphological transition from yeast to hypha. Therefore, our results revealed that suloctidil is a potential treatment for superficial biofilmassociated Candidal infections. However, detailed pharmacodynamics and pharmacological studies should be conducted in the future.

\section{MATERIALS AND METHODS}

\section{Organisms, media, and growth conditions}

This study utilized wild type strains of C. albicans, YEM30 and LC3, isolated from a patient with vaginal candidiasis from QiLu hospital. All strains were stored in $20 \%$ glycerol at $-80^{\circ} \mathrm{C}$ prior to the experiments. The frozen glycerol stock of the strain was regularly revived on YPD agar medium (1\% yeast extract, $2 \%$ tryptone, $2 \%$ glucose, and $2 \%$ agar). For the broth culture, strains were grown in YPD medium at $30^{\circ} \mathrm{C}$ with agitation (100 rpm). RPMI-1640 medium was buffered with morpholinepropanesulfonic acid buffer (Sigma-Aldrich, St. Louis, US) to $\mathrm{pH}$ 7. Stock solutions of suloctidil (Sigma-Aldrich, St. Louis, US) were prepared in dimethyl sulfoxide (DMSO, Sigma-Aldrich, St. Louis, US) and stored at $-20^{\circ} \mathrm{C}$ until further use.

\section{Biofilm development}

All strain cells were washed twice with sterile phosphate-buffered saline (PBS) and added to RPMI1640 medium at a density of $1 \times 10^{7}$ cell $/ \mathrm{mL}$. Biofilm experiments were performed in untreated 96-well cell culture plates. Briefly, $100 \mu \mathrm{L}$ of cell suspension was added to each well for $1.5 \mathrm{~h}$ of adhesion at $37^{\circ} \mathrm{C}$ with agitation $(75 \mathrm{rpm})$. The wells were washed twice with PBS to remove non-adherent cells. To allow the growth of biofilm, $200 \mu \mathrm{L}$ of fresh RPMI-1640 medium was added to each well. The plates were incubated in an orbital shaker for $24-48 \mathrm{~h}$ at $37^{\circ} \mathrm{C}$ with agitation $(75 \mathrm{rpm})$. At $24 \mathrm{~h}$ of 
incubation, the medium was aspirated, and the biofilms were washed twice with PBS, followed by the addition of $200 \mu \mathrm{L}$ of freshly prepared RPMI-1640 medium.

\section{Suloctidil susceptibility testing}

MICs of suloctidil were investigated in 96-well plates with RPMI-1640 medium in accordance with the broth microdilution method for the CLSI standard M27-A2 [15]. Stock solutions of suloctidil at $40 \mathrm{mg} / \mathrm{mL}$ were used for MIC studies. Then, $100 \mu \mathrm{L}$ two-fold dilutions of suloctidil (4-32 $\mu \mathrm{g} / \mathrm{mL})$ were added. Each cell suspension $\left(5 \times 10^{6} \mathrm{CFU} / \mathrm{mL}\right)$ was diluted (1:50 dilution, followed by $1: 20$ dilution) in RPMI-1640 medium to obtain twice the final inoculum size of $2.5 \times 10^{3} \mathrm{CFU} / \mathrm{mL}$. $100 \mu \mathrm{L}$ of inoculum was then added, and the plates were incubated for $48 \mathrm{~h}$ at $37^{\circ} \mathrm{C}$ with agitation $(75 \mathrm{rpm})$. $\mathrm{MIC}_{80}$ was expressed as the minimal concentration in the well that exhibited $20 \%$ growth of planktonic cells compared with that of the positive control wells. Growth was detected as turbidity $(492 \mathrm{~nm})$ relative to the uninoculated well using a microtiter plate reader. RPMI-1640 medium was used as a negative control, and overnight-cultured $C$. albicans was used as positive controls in the wells. Selected wells that contained $100 \mu \mathrm{L}$ of RPMI-1640 with 5\% DMSO and without suloctidil were set as the control.

\section{Effect of suloctidil on $C$. albicans biofilm formation and on pre-formed biofilms}

The biofilm formation assay was performed in 96-well plates as previously described, with slight modifications. The cell suspension was prepared in RPMI-1640 medium at a density of $1 \times 10^{7}$ cells $/ \mathrm{mL}$, dispensed into 96 -well plates $(100 \mu \mathrm{L}$ per well), and incubated for $1.5 \mathrm{~h}$ at $37^{\circ} \mathrm{C}$ with agitation $(75 \mathrm{rpm})$. After incubation, the cell suspensions were removed, and each well was washed twice with PBS to remove non-adherent cells. Two-fold dilutions of suloctidil in RPMI-1640 medium (100 $\mu \mathrm{L}$ per well) were added to each well. Then, the plates were further incubated for $24 \mathrm{~h}$. Similarly, 100 $\mu \mathrm{L}$ of RPMI-1640 medium that contained 5\% DMSO without suloctidil was added into the selected wells for the control.

To obtain preformed biofilms, the medium was aspirated after $48 \mathrm{~h}$ of incubation. Non-adherent cells were removed by washing the biofilms twice with sterile PBS. Briefly, $100 \mu \mathrm{L}$ of two-fold dilutions of suloctidil were added to the wells of prewashed biofilms. The controls were performed as previously described. Furthermore, 96well plates were incubated at $37^{\circ} \mathrm{C}$ for $24 \mathrm{~h}$.

The metabolic activity of biofilms was quantitatively determined by colorimetric XTT [2,3-bis-(2-methoxy-4nitro-5-sulfophenyl)-2H-tetraz- olium-5-carboxanilide sodium salt] reduction assay.

\section{Oxidative activity assay}

Biofilm growth activity was quantified via the colorimetric XTT reduction assay [16, 17]. XTT (Sigma-Aldrich, St. Louis, US) was dissolved in sterile PBS to obtain a $1 \mathrm{mg} / \mathrm{mL}$ XTT solution. The solution was sterilized and stored at $-70^{\circ} \mathrm{C}$ until use. Menadione solution (0.4 mM; Sigma-Aldrich, St. Louis, US) was prepared before use. Anhydrous D (+) glucose was dissolved in PBS at a final concentration of $200 \mathrm{mM}$. The XTT solution was thawed on ice and mixed with the menadione solution at a volume ratio of 20:1. Nonadherent cells were removed by washing the biofilms twice with sterile PBS. Then, $158 \mu \mathrm{L}$ of glucose, 40 $\mu \mathrm{L}$ of XTT, and $2 \mu \mathrm{L}$ of menadione were added to each well of the 96-well plates. The plates were covered with aluminum foil and incubated at $37^{\circ} \mathrm{C}$ for $3 \mathrm{~h}$. Thereafter, $100 \mu \mathrm{L}$ of the solution was transferred to new 96-well plates. The absorbance was measured at $492 \mathrm{~nm}$ using a microtiter plate reader.

\section{Time-kill curve}

The kinetics of the antibiofilm activity of the drugs was investigated using the time-kill test. As previously described, the medium was aspirated at $1.5 \mathrm{~h}$ adhesion, followed by the addition of $100 \mu \mathrm{L}$ of RPMI1640 medium. The two strains were treated with 100 $\mu \mathrm{L}$ of suloctidil at concentrations of $4,8,16$, and $32 \mu \mathrm{g} /$ $\mathrm{mL}$. The 96-well plates were covered with aluminum foil and incubated at $37^{\circ} \mathrm{C}$ with agitation $(75 \mathrm{rpm})$. At predetermined time points $(3,6,9,12,18,24 \mathrm{~h})$, the media from the corresponding wells were removed and washed twice with PBS. The metabolic activity of the biofilms was quantitatively determined by colorimetric XTT reduction assay.

Preformed biofilms were developed in 96-well plates as previously described. The medium was aspirated at $48 \mathrm{~h}$ of incubation. The biofilms were washed twice with PBS, followed by the addition of $100 \mu \mathrm{L}$ of fresh medium. Suloctidil was used at concentrations of 32, 64, 128 and $256 \mu \mathrm{g} / \mathrm{mL}$ for both strains. The $96-w e l l$ plates were covered with aluminum foil and incubated at $37^{\circ} \mathrm{C}$ with agitation $(75 \mathrm{rpm})$. Each well was aspirated and washed twice with PBS at predetermined time points $(0.5,1,3,6 \mathrm{~h})$. The metabolic activity of biofilms was quantified as previously described.

\section{Microscopic analyses}

C. albicans biofilms (YEM30) in the mature phase (48 h-old) with or without suloctidil $(64 \mu \mathrm{g} / \mathrm{mL})$ treatment were grown on glass cover slips in 6-well cell culture plates (Corning Inc., New York, US). The glass cover slips were placed in fixative $(3 \%$ glutaraldehyde 
in PBS) overnight. After rinsing with PBS and 30 min of immersion in $1 \%$ osmium tetroxide, the sample was dehydrated, dried, mounted, sputtered with gold, and visualized under a scanning electron microscope (SEM) (Sigma 300, Carl Zeiss Inc., Oberkochen, Germany).

\section{Hyphal growth test}

Previous studies have reported that hyphal growth is highly associated with $C$. albicans virulence and pathogenesis [18]. We performed the hyphal growth test to verify the antihyphal formation activity of suloctidil by visualizing the morphological changes in the strains. The hyphal growth assay was tested on the hyphal growth of C. albicans YEM30. C. albicans were developed for $0 \mathrm{~h}, 2 \mathrm{~h}$ and $4 \mathrm{~h}$ in different plates. Afterwards, cell suspensions were aspirated, and a total of $1 \mathrm{~mL}$ of fresh RPMI-1640 medium and $1 \mathrm{~mL}$ of suloctidil $(16 \mu \mathrm{g} /$ $\mathrm{mL})$ was added to the wells. The plates were incubated further for $12 \mathrm{~h}$ at $37^{\circ} \mathrm{C}$ with agitation $(75 \mathrm{rpm})$. Fresh RPMI-1640 medium with DMSO was used as a control. Thereafter, critical images were stored. All images are presented in Figure 4.

\section{Expression analysis of $C$. albicans hypha-specific genes by qRT-PCR}

The effect of sub $\mathrm{BIC}_{80}$ of suloctidil on the expression of hypha-specific genes $H W P 1, A L S 3$, and ECE1 was evaluated by quantitative real-time reverse transcription PCR (qRT-PCR). C. albicans YEM30 suspension was added to RPMI-1640 medium that contained hyphal inducer ( $5 \% \mathrm{FBS})$ at a concentration of $1 \times 10^{7}$ cell $/ \mathrm{mL}$. This test was performed in sterile culture plates (Thermo Fisher, Waltham, USA). First, $1 \mathrm{~mL}$ of cell suspension was transferred to culture plates and incubated for $1.5 \mathrm{~h}$ at $37^{\circ} \mathrm{C}$. After incubation, the cell suspensions were removed, $1 \mathrm{~mL}$ of suloctidil $(4 \mu \mathrm{g} / \mathrm{mL})$ was added, and $100 \mu \mathrm{l}$ of RPMI-1640 medium containing 5\% DMSO without suloctidil was used as the control. After $18 \mathrm{~h}$ of incubation at $37^{\circ} \mathrm{C}$, the plates were washed twice with PBS and transferred to microtubes.

The total RNA of the cells was investigated via the TRIzol method [19]. Following extraction, RNA concentration, purity, and quality were verified using a BioPhotometer plus (Eppendorf AG, Hamburg, Germany). The cDNA was synthesized using a PrimeScript ${ }^{\mathrm{TM}}$ RT Reagent Kit with gDNA Eraser (Takara, Kusatsu, Japan) in accordance with the manufacturer's instructions. Primers for the target (HWP1, ALS3, and ECE1) and housekeeping internal control (ACT1) genes (Table 2) were synthesized by BioCune (Shanghai, China). The cDNA template (100 $\mathrm{ng}$, gene-specific sense and antisense primers (400 nM), and SYBR ${ }^{\circledR}$ Premix Ex Tag (Takara, Kusatsu, Japan) were used in the reaction mixture per the manufacturer's instructions. The qRT-PCR was performed using a Mastercycler ${ }^{\circledR}$ ep realplex Real-time PCR system. The following parameters were used for qRT-PCR: initial denaturation at $95^{\circ} \mathrm{C}(3 \mathrm{~min})$, followed by 40 cycles of denaturation $\left(95^{\circ} \mathrm{C} / 5 \mathrm{~s}\right)$, annealing $\left(60^{\circ} \mathrm{C} / 30 \mathrm{~s}\right)$, extension $\left(75^{\circ} \mathrm{C} / 45 \mathrm{~s}\right)$, and melting-curve analysis starting from $95^{\circ} \mathrm{C}$ for $15 \mathrm{~s}, 60^{\circ} \mathrm{C}$ for $1 \mathrm{~min}$, and $95^{\circ} \mathrm{C}$ for $15 \mathrm{~s}$. The specificity of the primers was confirmed using melting curve analysis. The generated $\mathrm{C}_{\mathrm{t}}$ values of target genes were normalized to the $\mathrm{C}_{\mathrm{t}}$ value of the housekeeping ACT1 gene. Relative expression fold changes were evaluated by the $\Delta \Delta \mathrm{C}_{\mathrm{t}}$ method using a previously published formula [19].

\section{Murine vaginal candidiasis model}

The murine vaginal candidiasis model was established with Kunming mice following a previously described method with minor modifications [20-22]. Fifty-three female Kunming mice (28-32 g body weight) were used at 7 to 8 weeks of age. The mice were randomly assigned to 2 groups with five mice each: either vehicle or suloctidil and were maintained under a pseudoestrus condition by subcutaneous injection of $0.2 \mathrm{mg}$ of estradiol benzoate (every other day, CEN'S, Hangzhou, China) 7 days prior to infection and until the completion of the study. A C. albicans suspension $\left(1 \times 10^{7}\right.$ cells $\left./ \mathrm{mL}\right)$ in $20 \mu \mathrm{L}$ of PBS was administered to the vaginas of the mice. The filaments in the mouse vaginas were observed after 7 days of infection. Intravaginal treatment with $50 \mu \mathrm{L}$ of $256 \mu \mathrm{g} /$ $\mathrm{mL}$ suloctidil was administered into the vaginas of mice every day until day +21 . PBS was used as a control. After various treatment times, the mice were sacrificed and the vaginal lumen was thoroughly washed and scraped with $1 \mathrm{~mL}$ of PBS. To determine the fungal load in the vagina, $100 \mu \mathrm{L}$ of lavage fluid from each mouse was plated on YPD agar plus ceftriaxone $(50 \mu \mathrm{g} / \mathrm{mL}$, Roche, Basel, Switzerland). CFUs were then evaluated.

\section{Histological analysis}

The mice vaginas were removed, stored in the same fixative solution ( $10 \%$ buffered formalin), and embedded in paraffin. The vaginal tissue was sliced into 5 - $\mu \mathrm{m}$ thick sections for hematoxylin and eosin staining (HE) and examined by light microscopy. Inflammatory cell infiltrates (Polymorphonuclear leukocytes and mononuclear cells) in the antrum and body were graded as described: 0 , none; 1 , some infiltrates; 2 , mild infiltrates (few aggregates in submucosa and mucosa); 3 , moderate infiltrates (several aggregates in submucosa and mucosa); 4, marked infiltrates (many large aggregates in submucosa and mucosa); 5, nearly the entire mucosa contained a dense infiltrate; and 6 , the entire mucosa contained a dense infiltrate [23]. 


\section{Statistical analysis}

All assays were performed in triplicate, and each experiment was repeated thrice on independent days unless otherwise stated. A total of 5 repetitions per plate were performed for the murine vaginal candidiasis model experiment. All obtained results were expressed as the mean \pm standard deviation. All data sets for the comparison of treated and control groups were analyzed by one-way ANOVA. All analyses were conducted with GraphPad Software (GraphPad Prism ${ }^{\circledR}$ Version 6.0c, La Jolla, CA, USA) at a $95 \%$ confidence level.

\section{Author contributions}

ZYB and SYD conceived and designed the study. ZBN, LJC, WYJ, CPX, WXH, CJF, LLD, HXY, CQ, XX and DJL performed the experiments. ZBN and SYD wrote the paper. ZYB reviewed and edited the manuscript. All authors read and approved the manuscript.

\section{ACKNOWLEDGMENTS}

The authors acknowledge Mr. Shuping Ren, Shandong University, for assistance with feeding the mice.

\section{CONFLICTS OF INTEREST}

All authors have no conflicts of interest.

\section{FUNDING}

The present research was supported by the National Natural Science Foundation of China (Nos. 81471991, 81671978, 81401696, and 81371781, 81372680, 81571986), the National Basic Research Program of China (973 Program 2012CB911202), the National Natural Science Foundation of Shandong Province (ZR2011HL010) and the National Basic Research Program of Shandong Province (2015GSF118147).

\section{REFERENCES}

1. Haque F, Alfatah M, Ganesan K, Bhattacharyya MS. Inhibitory effect of sophorolipid on Candida albicans biofilm formation and hyphal growth. Sci Rep. 2016; 6:23575

2. Sardi JC, Scorzoni L, Bernardi T, Fusco-Almeida AM, Mendes Giannini MJ. Candida species: current epidemiology, pathogenicity, biofilm formation, natural antifungal products and new therapeutic options. J Med Microbiol. 2013; 62:10-24.

3. Chandra J, Kuhn DM, Mukherjee PK, Hoyer LL, McCormick T, Ghannoum MA. Biofilm formation by the fungal pathogen Candida albicans: development, architecture, and drug resistance. J Bacteriol. 2001; 183:5385-5394.

4. Wang B, You J, King JB, Cai S, Park E, Powell DR, Cichewicz RH. Polyketide glycosides from Bionectria ochroleuca inhibit Candida albicans biofilm formation. J Nat Prod. 2014; 77:2273-2279.

5. Falagas ME, Betsi GI, Athanasiou S. Probiotics for prevention of recurrent vulvovaginal candidiasis: a review. J Antimicrob Chemother. 2006; 58:266-272.

6. An D, Wang X, Li J, Jiang S, Ma X, Zhang H, Shi H, Sun $\mathrm{H}$, Ye L, Li J. The activity of fungichromin against the formation of Candida albicans biofilm. Biol Pharm Bull. 2016; 39:1948-1954.

7. Kathwate GH, Shinde RB, Karuppayil SM. Antiepileptic drugs inhibit growth, dimorphism, and biofilm mode of growth in human pathogen Candida albicans. Assay Drug Dev Tech. 2015; 13:307-312.

8. Matsubara VH, Wang Y, Bandara HM, Mayer MP, Samaranayake LP. Probiotic lactobacilli inhibit early stages of Candida albicans biofilm development by reducing their growth, cell adhesion, and filamentation. Appl Microbiol Biotechnol. 2016; 100:6415-6426.

9. Kojic EM, Darouiche RO. Candida infections of medical devices. Clin Microbiol Rev. 2004; 17:255-267.

10. Ramage G, Martinez JP, Lopez-Ribot JL. Candida biofilms on implanted biomaterials: a clinically significant problem. FEMS Yeast Res. 2006; 6:979-986.

11. Alcazar-Fuoli L, Mellado E. Current status of antifungal resistance and its impact on clinical practice. Br J Haematol. 2014; 166:471-484.

12. Panwar R, Pemmaraju SC, Sharma AK, Pruthi V. Efficacy of ferulic acid encapsulated chitosan nanoparticles against Candida albicans biofilm. Microb Pathog. 2016; 95:21-31.

13. Boeynaem J, Coevorde A, Demolle D. Stimulation of prostacyclin prostacyclin production in blood vessels by the antithrombotic drug. Biochem Pharmacol. 1987; 36:1629-1635.

14. Butts A, DiDone L, Koselny K, Baxter BK, ChabrierRosello Y, Wellington M, Krysan DJ. A repurposing approach identifies off-patent drugs with fungicidal cryptococcal activity, a common structural chemotype, and pharmacological properties relevant to the treatment of cryptococcosis. Eukaryot Cell. 2013; 12:278-287.

15. Hamza OJ, van den Bout-van den Beukel CJ, Matee MI, Moshi MJ, Mikx FH, Selemani HO, Mbwambo ZH, Van der Ven AJ, Verweij PE. Antifungal activity of some Tanzanian plants used traditionally for the treatment of fungal infections. J Ethnopharmacol. 2006; 108:124-132.

16. Silva WJ, Seneviratine J, Parahitiyawa N, Rosa EA, Samaranayake LP. Improvement of XTT assay performance for studies involving Candida albicans biofilms. Braz Dent J. 2008; 19:364-369. 
17. Jin Y, Samaranayake LP, Samaranayake Y, Yip HK. Biofilm formation of Candida albicans is variably affected by saliva and dietary sugars. Arch Oral Biol. 2004; 49:789-798.

18. Gulati M, Nobile CJ. Candida albicans biofilms: development, regulation, and molecular mechanisms. Microbes Infect. 2016; 18:310-321.

19. Chang S, Chen W, Yang J. Another formula for calculating the gene change rate in real-time RT-PCR. Mol Biol Rep. 2009; 36:2165-2168.

20. Fidel PL, Cutright JJ, Tait L, Sobel JD. A murine model of Candida glabrata vaginitis. J Infect Dis. 1996; 173:425-31.

21. Pietrella D, Angiolella L, Vavala E, Rachini A, Mondello F, Ragno R, Bistoni F, Vecchiarelli A. Beneficial effect of Mentha suaveolens essential oil in the treatment of vaginal candidiasis assessed by real-time monitoring of infection. BMC Complement Altern Med. 2011; 11:18.

22. Harriott MM, Lilly EA, Rodriguez TE, Fidel PL Jr, Noverr MC. Candida albicans forms biofilms on the vaginal mucosa. Microbiology. 2010; 156:3635-3644.

23. Shan Y, Lu X, Han Y, Li X, Wang X, Shao C, Wang L, Liu Z, Tang W, Sun Y, Jia J. Helicobacter pylori outer membrane protein 18 (Hp1125) is involved in persistent colonization by evading interferon- gamma signaling. Biomed Res Int. 2015; 2015:571280.

24. Jia W, Zhang H, Li C, Li G, Liu X, Wei J. The calcineruin inhibitor cyclosporine a synergistically enhances the susceptibility of Candida albicans biofilms to fluconazole by multiple mechanisms. BMC Microbiol. 2016; 16:113.

25. Birse CL, Irwin MY, Fonzi WA, Sypherdonzi PS. Cloning and characterization of ECE1, a gene expressed in association with cell elongation of the dimorphic pathogen Candida albicans. Infect Immun. 1993; 61:3648-3655.

26. Zhou Y, Wang G, Li Y, Liu Y, Song Y, Zheng W, Zhang N, $\mathrm{Hu} \mathrm{X}$, Yan S, Jia J. In vitro interactions between aspirin and amphotericin B against planktonic cells and biofilm cells of Candida albicans and C. parapsilosis. Antimicrob Agents Chemother. 2012; 56:3250-3260.
27. Piccioni F, Roman BR, Fischbeck KH, Taylor JP. A screen for drugs that protect against the cytotoxicity of polyglutamine-expanded androgen receptor. Human Mol Genet. 2004; 13:437-446.

28. Nobile CJ, Johnson AD. Candida albicans biofilms and human disease. Ann Rev Microbiol. 2015; 69:71-92.

29. Rooney PJ, Klein BS. Linking fungal morphogenesis with virulence. Cell Microbiol. 2002; 4:127-137.

30. Li D. Temporal expression of the Candida albicans genes CHK1 and CSSK1, adherence, and morphogenesis in a model of reconstituted human esophageal epithelial candidiasis. Infect Immun. 2002; 70:1558-1565.

31. Ding X, Liu Z, Su J, Yan D. Human serum inhibits adhesion and biofilm formation in Candida albicans. BMC Microbiol. 2014; 14:80

32. Sundstrom P. Adhesion in Candida spp. Cell Microbiol. 2002; 4:461-469.

33. Chatzimoschou A, Simitsopoulou M, Antachopoulos C, Walsh TJ, Roilides E. Differential effects of antifungal agents on expression of genes related to formation of Candida albicans biofilms. Mycoses. 2016; 59:43-47.

34. Nobile CJ, Schneider HA, Nett JE, Sheppard DC, Filler SG, Andes DR, Mitchell AP. Complementary adhesin function in C. albicans biofilm formation. Curr Biology. 2008; 18: 1017-1024.

35. Finke JS, Xu W, Huang D, Hill EM, Desai JV, Woolford CA, Nett JE, Taff H, Norice CT, Andes DR, Lanni F, Mitchell AP. Portrait of Candida albicans adherence regulators. PLoS Pathog. 2012; 8:e1002525.

36. Tronchin G, Pihet M, Lopes-Bezerra LM, Bouchara JP. Adherence mechanisms in human pathogenic fungi. Med Mycol. 2008; 46:749-772.

37. Desai JV, Mitchell AP. Candida albicans biofilm development and its genetic control. Microbiol Spectr. $2015 ; 3$. 